\title{
Comprehensive genomic analysis of Bacillus subtilis 9407 reveals its biocontrol potential against bacterial fruit blotch
}

\author{
Xiaofei Gu', Qingchao Zeng ${ }^{2}$, Yu Wang ${ }^{3}$, Jishun $\mathrm{Li}^{4}$, Yu Zhao ${ }^{1}$, Yan $\mathrm{Li}^{1}$ and Qi Wang ${ }^{1 *}$
}

\begin{abstract}
Bacillus subtilis, a plant-beneficial bacterial species exhibiting good biocontrol capabilities, has been widely used in agricultural production. The endophytic strain 9407 can efficiently control bacterial fruit blotch (BFB) caused by the gram-negative bacterium Acidovorax citrulli. However, the mechanism underlying its biocontrol ability remains poorly understood. Given the genomic diversity of B. subtilis, strain 9407 was sequenced and assembled in this study to determine the genome information associated with its biocontrol traits. A combination of core genome phylogenetic analysis and average nucleotide identity (ANI) analysis demonstrated that the 9407 strain belonged to B. subtilis. Various functional genes related to biocontrol traits, i.e., biofilm formation, motility, pathogen inhibition, plant growth promotion, and induction of systemic resistance, were identified in B. subtilis 9407 . Four secondary metabolite biosynthesis gene clusters with antibacterial ability were also found in the B. subtilis 9407 genome, including newly identified subtilosin A, bacilysin, and bacillaene, and the previously reported surfactin. Mutants lacking $s b o A$ or bacG, which are defective in synthesizing subtilosin A or bacilysin, showed decreased inhibitory activity against $A$. citrulli $\mathrm{MH} 21$, and the triple mutant with deleted $s b \circ A$, bacG, and $s r f A B$ almost completely lost its inhibitory activity. The biofilm formation and swarming motility of the sboA and bacG mutants also decreased, in turn leading to decreased colonization on melon roots and leaves. Under greenhouse conditions, the biocontrol efficacy of the sboA and bacG mutants against BFB on melon leaves decreased by 21.4 and 32.3\%, respectively. Here, we report a new biocontrol pathway of B. subtilis 9407 against BFB, in which subtilosin A and bacilysin contributed to the biocontrol efficacy by improving antibacterial activity and colonization ability of the strain. The comprehensive genomic analysis of B. subtilis 9407 improves our understanding of the biocontrol mechanisms of B. subtilis, providing support for further research of its biocontrol mechanisms and field applications.
\end{abstract}

Keywords: Bacillus subtilis, Genomic analysis, Biocontrol mechanism, Bacterial fruit blotch, Subtilosin A, Bacilysin

\section{Background}

Bacillus subtilis has been widely used in agricultural production due to its environmental safety, straightforward industrial production, and good biocontrol efficacy (Wang et al. 2020). The development of genome sequencing technology and bioinformatic analysis have made it convenient to obtain genomic information on B. subtilis,

\footnotetext{
* Correspondence: wangqi@cau.edu.cn

'Department of Plant Pathology, MOA Key Lab of Pest Monitoring and Green Management, College of Plant Protection, China Agricultural University, Beijing 100193, China

Full list of author information is available at the end of the article
}

allowing us to comprehensively understand its life activities (Moszer 1998). Genomic comparison of different $B$. subtilis strains can elucidate the genetic variation, evolutionary classification, and genomic diversity of this species (Rahimi et al. 2018). To date, the genome sequences of 389 strains of $B$. subtilis have been deposited in the National Center for Biotechnology Information (NCBI) genome assembly database. These genomic data have revealed important information about the development, sporulation, and metabolism of B. subtilis strains (Kunst et al. 1997), and can be further used to obtain

(c) The Author(s). 2021 Open Access This article is licensed under a Creative Commons Attribution 4.0 International License, which permits use, sharing, adaptation, distribution and reproduction in any medium or format, as long as you give appropriate credit to the original author(s) and the source, provide a link to the Creative Commons licence, and indicate if changes were made. The images or other third party material in this article are included in the article's Creative Commons licence, unless indicated otherwise in a credit line to the material. If material is not included in the article's Creative Commons licence and your intended use is not permitted by statutory regulation or exceeds the permitted use, you will need to obtain permission directly from the copyright holder. To view a copy of this licence, visit http://creativecommons.org/licenses/by/4.0/. 
new information related to their biocontrol traits (Sulthana et al. 2019; Franco-Sierra et al. 2020). In particular, the genomic sequence of the well-known model organism B. subtilis strain 168 has allowed the formulation of useful inferences for studies of other B. subtilis strains (Barbe et al. 2009).

Accumulating evidence indicates that B. subtilis possesses biocontrol traits, including plant colonization, pathogen inhibition, and plant growth promotion abilities, and activation of induced systemic resistance (Hashem et al. 2019). In B. subtilis, motility towards plant roots and biofilm formation on the root surface are crucial for its colonization of plant roots and biocontrol efficacy against plant pathogens (Gao et al. 2013; Allard-Massicotte et al. 2016; Al-Ali et al. 2018). The production of active substances is an important indicator for assessing the biocontrol efficacy of a beneficial strain (Zeriouh et al. 2014; Gao et al. 2016). B. subtilis produces various substances with broadspectrum antibacterial activity, including lipopeptide antibiotics, bacteriocins, and antibacterial proteins (Stein 2005). Importantly, it has been suggested that different antibacterial substances can act synergistically to inhibit phytopathogen growth (Koumoutsi et al. 2004; Alanjary and Medema 2018). Furthermore, the production of phytohormones, siderophores, lipopeptides, volatile compounds, and phytases allows B. subtilis to promote plant growth and induce plant immune responses (Franco-Sierra et al. 2020).

B. subtilis strain 9407, isolated from healthy apple fruit, has exhibited broad-spectrum antimicrobial activities (Fan et al. 2017a). Previously, we found that $B$. subtilis 9407 controls bacterial fruit blotch (BFB) through surfactin-mediated antibacterial activity (Fan et al. 2017b). BFB is a serious melon disease caused by Acidovorax citrulli and poses a serious threat to the melon industry (Bahar et al. 2008; Adhikari et al. 2017; Rahimi-Midani and Choi 2020). It is characterized by symptoms such as water-soaked disease spots. The main control strategy against BFB is the application of antibiotics and chemicals; therefore, more effective and environmentally-friendly control strategies are urgently needed (Rahimi-Midani and Choi 2020). B. subtilis, as a well-known environmentally-friendly biocontrol bacterial species, can exert its biocontrol effects via several pathways (Fira et al. 2018; Hashem et al. 2019). Although we have confirmed that B. subtilis 9407 controls BFB by producing surfactin, its biocontrol mechanism against BFB remains poorly understood. The objective of this study was to shed light on the underlying biocontrol mechanism of B. subtilis 9407 , especially those pathways that could effectively control BFB. We performed a comprehensive genome analysis of B. subtilis 9407 to reveal the biocontrol mechanism of this specific strain, and to determine which substances play a direct role against BFB. The results will contribute to the development of new biocontrol agents with original modes of action against specific plant diseases.

\section{Results}

\section{General genome description of $B$. subtilis 9407}

In this study, we sequenced the genome of $B$. subtilis 9407 to explore its biocontrol mechanism. Genomic assembly of B. subtilis 9407 produced 16 scaffolds, with an $\mathrm{N}_{50}$ of $2,111,374 \mathrm{bp}$. The whole-genome sequence of $B$. subtilis 9407 is $4,062,615$ bp in length with a $\mathrm{G}+\mathrm{C}$ content of 43.7\% (Table 1 and Additional file 1: Figure S1). The number of predicted protein-coding genes in $B$. subtilis 9407 is 4033. Among these, 2853 were assigned a putative function, and 1180 were predicted to encode hypothetical proteins. The protein-coding genes had an average length of $884 \mathrm{bp}$ and accounted for $89.1 \%$ of the genomic sequence. A total of 79 tRNA-coding genes and 9 rRNA genes were predicted in the chromosome sequence.

\section{Phylogenetic analysis of $B$. subtilis 9407}

As a molecular marker, the $16 \mathrm{~S}$ rRNA gene has been widely used for strain identification, but microbial taxonomies based on 16S rRNA gene relationships still have limitations, including low phylogenetic resolution. Phylogeny construction based on the core genome has progressed in recent years towards a standardized bacterial taxonomy (Parks et al. 2018). In this study, a phylogenetic tree was constructed using core genome analysis to understand the evolutionary relationships of B. subtilis strain 9407. A phylogenetic tree of 16 Bacillus genomes was constructed based on the concatenation of 662 single-copy core genes present in all genomes using the maximum likelihood (ML) method and rooted in Paenibacillus polymyxa M1. As shown in Fig. 1, B. subtilis 9407 is in the same clade with other B. subtilis strains and a sister group of B. subtilis SEM-9.

Table 1 General genome features of B. subtilis 9407

\begin{tabular}{ll}
\hline Category & B. subtilis $\mathbf{9 4 0 7}$ \\
\hline Genome size (bp) & $4,062,615$ \\
G+C content (\%) & 43.7 \\
Protein-coding genes & 4033 \\
Total gene length (bp) & $3,615,252$ \\
Average gene length (bp) & 884 \\
Gene length/genome (\%) & 89.1 \\
Genes with assigned function & 2853 \\
tRNA & 79 \\
rRNA & 9 \\
\hline
\end{tabular}




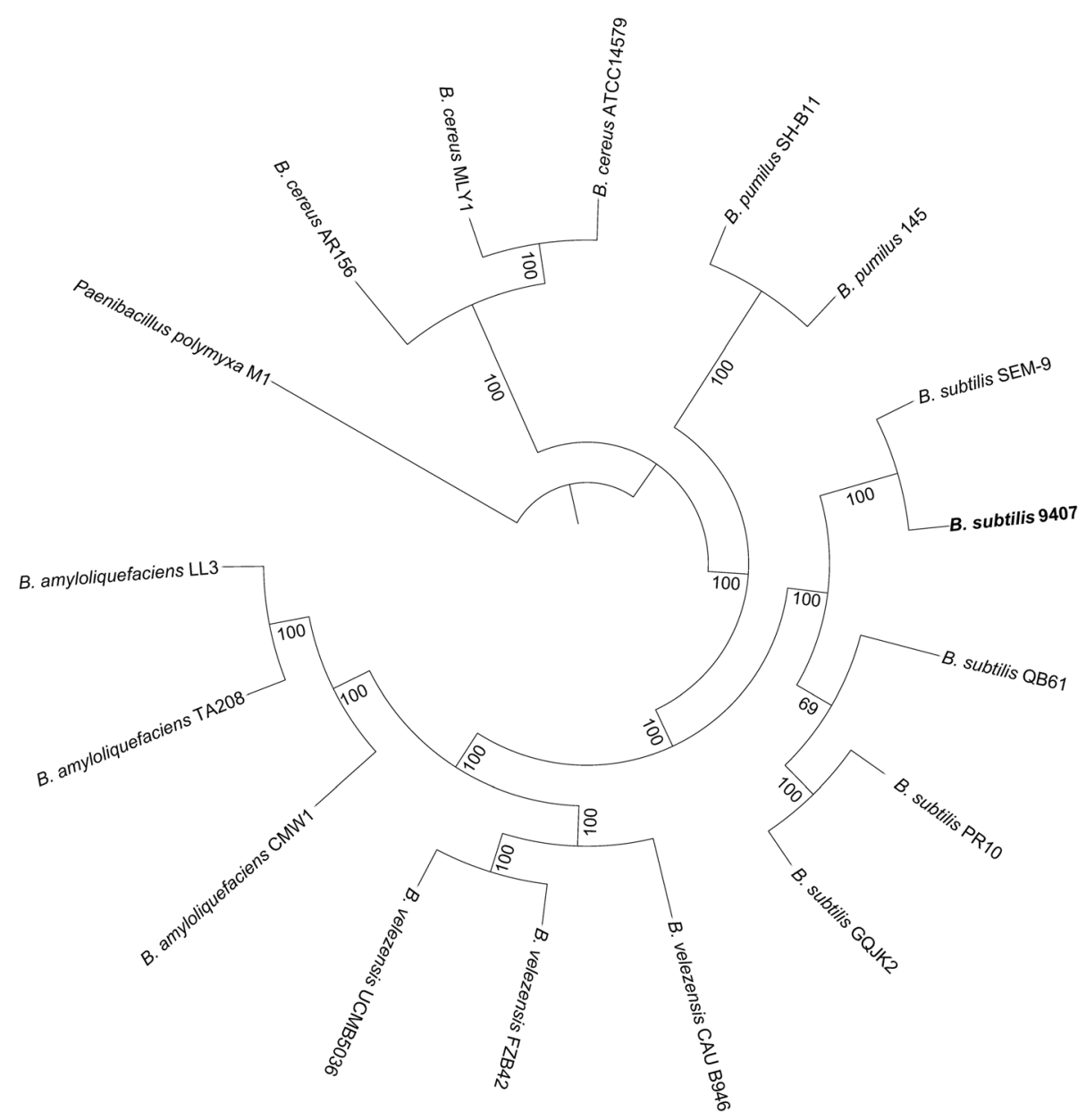

Fig. 1 Phylogenetic tree showing the relationship of the sequenced Bacillus spp. strains. The tree was generated based on the 662 single-copy core genes using RAxML 8.2.10. P. polymyxa M1 was used as the out-group. Percent bootstrap values (from 100 replicates) are indicated at the nodes

Average nucleotide identity (ANI) is the average identity value calculated from a pair-wise comparison of homologous sequences between two genomes; this indicator is frequently used in species definition (Lee et al. 2016). In this study, we conducted a heatmap analysis based on the ANI values of different strains to confirm the findings of our phylogenetic analysis. The ANI values of representative Bacillus strains are summarized in Fig. 2. B. subtilis 9407 and other B. subtilis strains showed ANI values of $>98 \%$, suggesting that they are the same species. A pan-genome analysis indicated that the selected B. subtilis strains contain 3153 common genes, comprising 92.7-99.3\% of all genes (Fig. 3). To intuitively illustrate the results above, we performed a comparative genomic analysis using BLAST Ring Image Generator (BRIG) software to evaluate synteny between B. subtilis strains 9407 and 168 (Sulthana et al. 2019). The results showed that there was a high genomic similarity between these two strains, suggesting that their genetic information is very similar (Additional file 1: Figure S2).

\section{Potential functional genes involved in biocontrol traits of B. subtilis 9407}

Bacillus harbors various functional genes associated with biocontrol traits, thereby ensuring its biocontrol efficacy (Ashwini and Srividya 2014). In this study, potential functional genes related to biocontrol traits of B. subtilis 9407 were analyzed based on whole-genome annotation and pan-genome analysis results. The results showed that $B$. subtilis 9407 possesses several functional genes involved in biofilm formation, motility, pathogen inhibition, plant growth promotion, and induced systemic resistance, sharing $91-100 \%$ identity and $98-100 \%$ genome coverage with $B$. subtilis 168 (Additional file 2: Tables S1 and S2). Genes related to biofilm formation, 


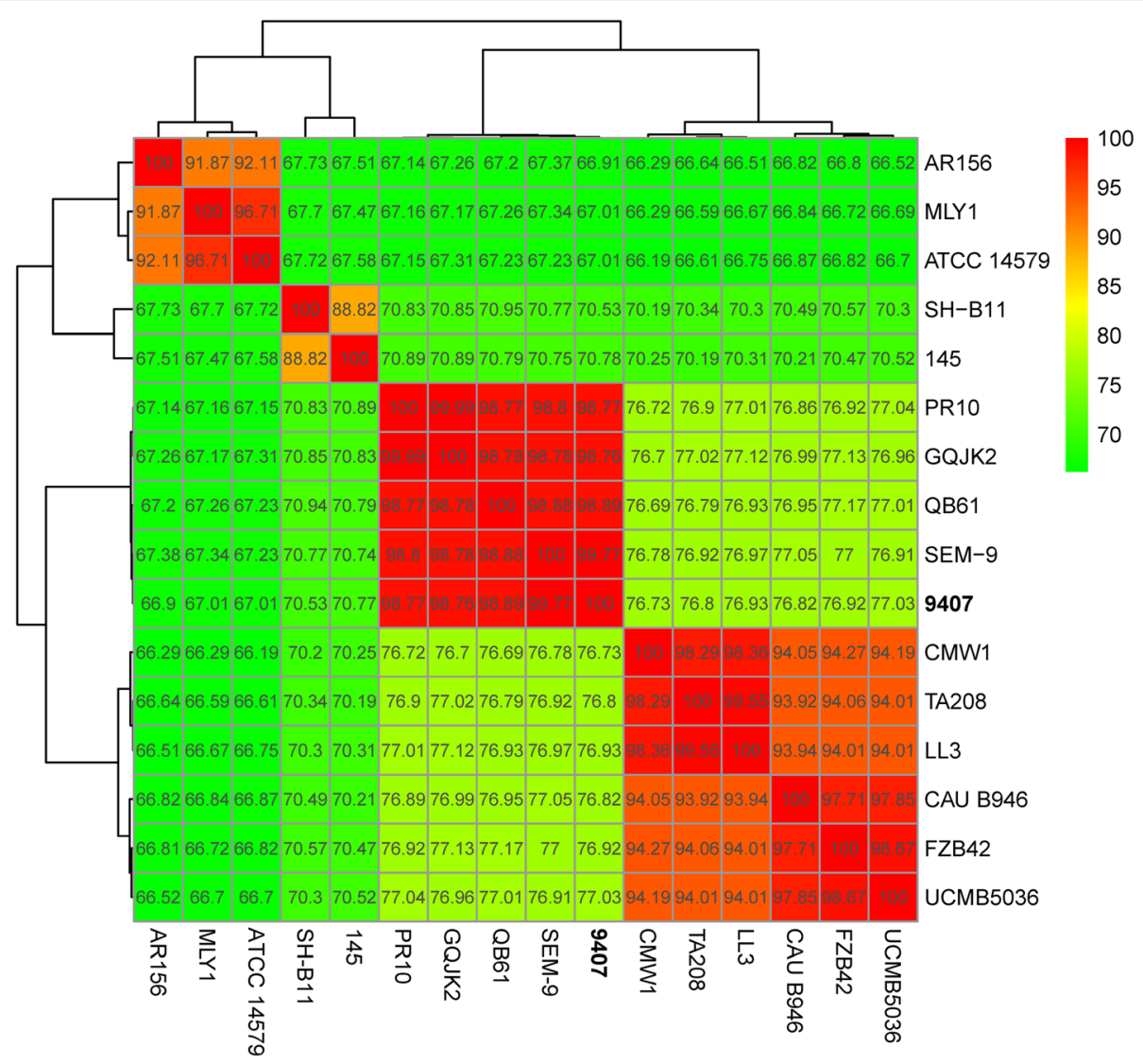

Fig. 2 Heat-map of Average Nucleotide Identity (ANI) values amongst different Bacillus strains. The numbers represent the size of ANI values

motility, and flagellum biosynthesis were found in $B$. subtilis 9407, including biofilm synthetic genes (eps operon, tapA-sip W-tasA operon, blsA, pgs operon), regulatory genes $(\operatorname{spoOA} a b r B, \sin R, \sin I$, etc.), and flagellum biosynthesis genes (cheY, motA, motB, flg and fli operon), which are well known to be involved in colonization ability. Several genes were found to be involved in the production of volatile organic compounds (VOCs), phytohormones, and siderophore bacillibactin, suggesting that $B$. subtilis 9407 has the potential to promote plant growth. For example, $y s n E, y w k B, p h y C$, and $d h b$ operon are involved in the synthesis of indole-3acetic acid (IAA), auxin, phytase, bacillibactin, respectively. Various genes encoding proteins associated with induced plant systemic resistance, i.e., srf operon, als operon, and $b d h A$ encoding surfactin, acetoin, and 2, 3butanediol, respectively, were detected in B. subtillis 9407. TasA and lipopeptide surfactin are also well known for their antimicrobial activity. Synthetic genes of other antibacterial and antifungal substances were also found, such as pps operon encoding fengycin, bac encoding bacilysin, sbo $A$ encoding subtilosin $\mathrm{A}$, and $p k s$ encoding bacillaene. These findings demonstrate that $B$. subtilis 9407 has the potential to colonize plants, inhibit pathogens, promote plant growth, and induce plant systemic resistance.

\section{Prediction of biosynthesis gene clusters in the genome sequence of $B$. subtillis 9407}

Various widely reported secondary metabolites produced by $B$. subtilis are beneficial to the survival of this bacterial species in the complex and changeable natural environment (Stein 2005). In this study, six biosynthesis gene clusters (BGCs) were found in the genome of B. subtilis 9407, including four nonribosomal peptide synthetases (bacillibactin, bacilysin, fengycin, and surfactin), one trans-acyl transferase polyketide synthetase (bacillaene), and one sactipeptide (subtilosin A), all sharing a high degree of sequence similarity with those of B. subtilis 168 (Table 2 and Additional file 1: Figure S3). The amino acid sequence identity of each gene in the BGCs between B. subtilis strains 9407 and 168 was $95-100 \%$. However, bacE within the bacilysin cluster in B. subtilis 9407 seemed to be partially missing, and an additional gene of unknown function was found in the bacillaene cluster of B. subtilis 9407 , but not in that of B. subtilis 


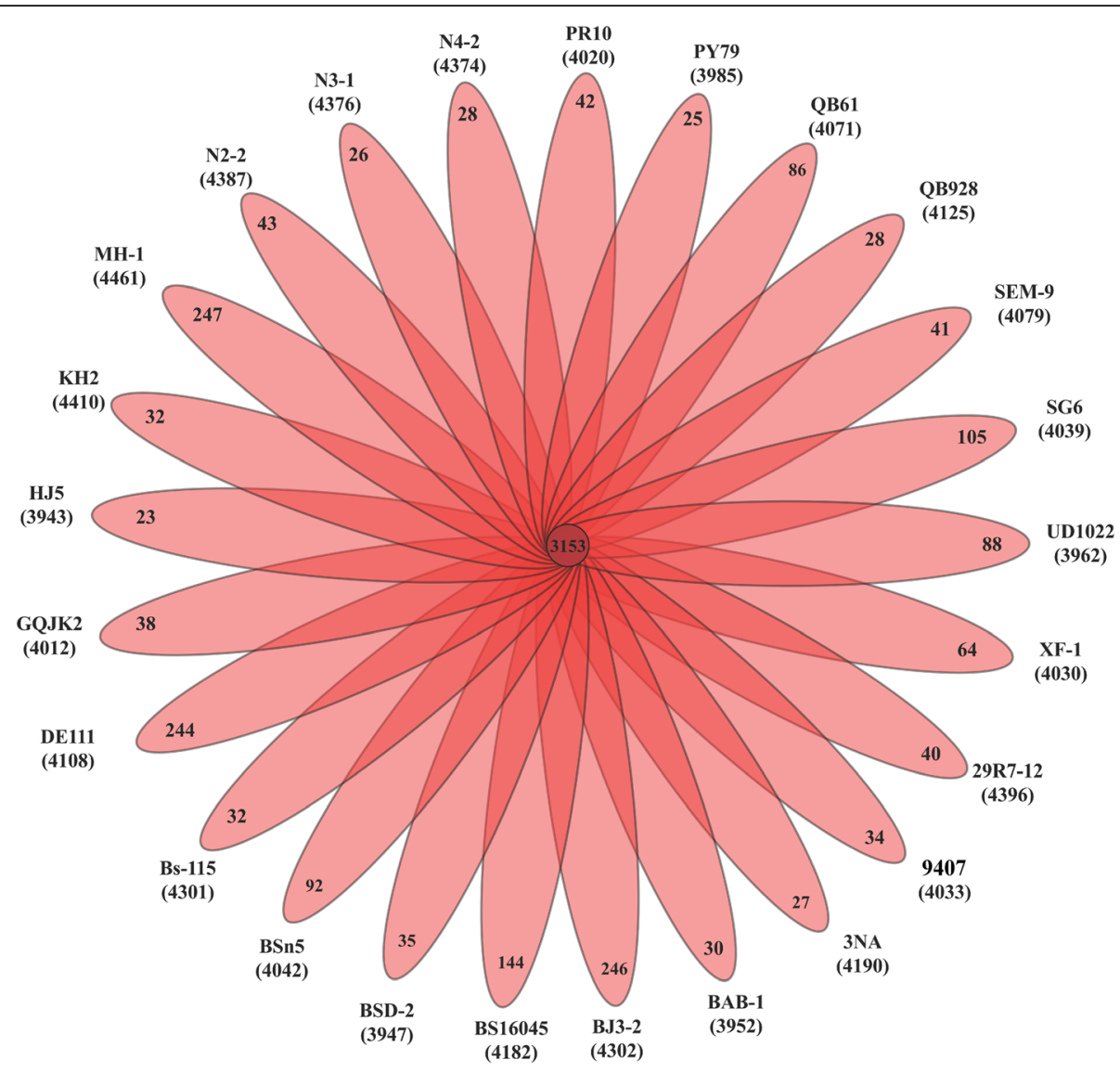

Fig. 3 Pan-genome analysis of B. subtilis 9407 and 24 other B. subtilis strains. Flower plot showing numbers of species-specific genes commonly found in each genome of each species (in the petals), and B. subtilis core orthologous gene number (in the center)

168. Whether these differences were caused by sequencing and assembling errors or natural variation, and whether they can influence the production of substances related to the life activity of $B$. subtilis 9407 remains unknown. Subtilosin A, bacilysin, bacillaene, and surfactin have been reported to possess antibacterial activity, suggesting that multiple substances may be involved in the antagonism of B. subtilis 9407 to A. citrulli $\mathrm{MH} 21$.

\section{Subtilosin $A$ and bacilysin participate in biocontrol efficacy of $B$. subtilis 9407 against BFB}

The synthetic genes $\operatorname{srf} A B$, sbo $A$, and $b a c G$ are essential for the synthesis of surfactin, subtilosin A, and bacilysin, respectively (Zheng et al. 2000; Stein 2005; Rajavel et al. 2013). Our previous studies showed that a lack of $\operatorname{srf} A B$ decreases surfactin production and colonization ability of B. subtilis 9407, thereby decreasing its biocontrol efficacy against BFB (Fan et al. 2017b). To verify whether

Table 2 Comparison of predicted BGCs between genomes of the B. subtilis strains 9407 and 168

\begin{tabular}{lllllll}
\hline Metabolites & Type & Clusters in $\mathbf{9 4 0 7}$ & Size (kb) & Clusters in 168 & Identity (\%) & Bioactive spectrum \\
\hline Bacillibactin & NRPS & dhbABCEF, besA & 49.7 & dhbABCEF, besA & $99-100$ & Microbial competitors \\
Subtilosin A & Sactipeptide & sboA, albABCDEFG & 21.6 & sboA, albABCDEFG & $98-100$ & Bacteria \\
Bacilysin & NRPS & bacABCDEFG & 49.7 & bacABCDEFG & $95-100$ & Bacteria, yeasts, and fungi \\
Fengycin & PKS/NRPS & ppsABCDE & 82.1 & ppsABCDE & $96-98$ & Filamentous fungi \\
Bacillaene & PKS/NRPS & baeABCDEGHILMNRS, acpKK & 114.8 & baeABCDEGHIJMNRS, acpK & $98-100$ & Bacteria \\
Surfactin & NRPS & srfAABCD & 65.4 & srfAABCD & $98-100$ & Virus, mycoplasma, and \\
& & & & & tumor \\
Sublancin 168 & Glycocin & Not present & - & sunATI, bdbAB & 0 & Gram-positive bacteria \\
Sporulation killing factor & Sactipeptide & Not present & - & skfABCEFGH & 0 & Bacteria \\
\hline
\end{tabular}


subtilosin A and bacilysin are involved in the biocontrol of B. subtilis 9407 against $A$. citrulli MH21, the causal agent of BFB, we constructed mutant strains by deleting sboA or bacG. The sboA and bacG mutants showed weaker antimicrobial activity to $A$. citrulli MH21 than the wild-type strains. In the triple mutant, showing srf $A B$, sboA, and bacG deletion, inhibitory activity against $A$. citrulli $\mathrm{MH} 21$ was almost completely lost (Fig. 4), suggesting that subtilosin A, bacilysin, and surfactin have a synergistic effect in inhibiting $A$. citrulli MH21. Compared with the wild-type strain, the $s b o A$ and bacG mutants simultaneously showed weaker biofilm formation (Fig. 5a, b) and swarming ability (Fig. 5c, d), suggesting that subtilosin A and bacilysin may affect the colonization ability of B. subtilis 9407 . Subsequent colonization experiments verified the hypothesis that sboA and bacG mutants would show decreased colonization ability (Fig. 5e). Colonization of the $s b o A$ mutant on melon roots and leaves decreased by 21.11 and $30.97 \%$, respectively, whereas that of the bacG mutant decreased by 23.94 and $32.48 \%$, respectively. The biocontrol efficacy of $s b o A$ and $b a c G$ mutants against BFB decreased by 21.4 and $32.2 \%$, respectively, under greenhouse conditions (Table 3 and Fig. 6). In summary, subtilosin A and bacilysin affected the biocontrol efficacy of B. subtilis 9407 against BFB by influencing its antibacterial activity and colonization ability.

\section{Discussion}

Microbial biocontrol strategies against BFB have been widely reported. For instance, B. subtilis R14, B. megaterium pv. cerealis RAB7, B. pumilus C116, and Bacillus sp. MEN2 show antibiosis against $A$. citrulli by producing bioactive compounds that are partially characterized as lipopeptides (Santos et al. 2006). B. amyloliquefaciens 54 significantly controls BFB by increasing the expression of an important defense-related gene, PR1 (Jiang et al. 2015). Bacteriophages effectively control BFB by translocating from soil to leaf tissue and killing $A$. citrulli (Rahimi-Midani and Choi 2020). Bacillus strains exert biocontrol efficacy through several biocontrol mechanisms (Fira et al. 2018; Hashem et al. 2019). In

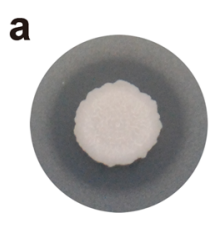

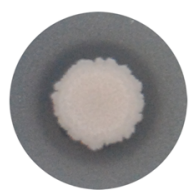

sboA

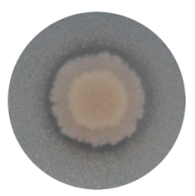

bacG

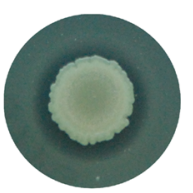

sifAB

b

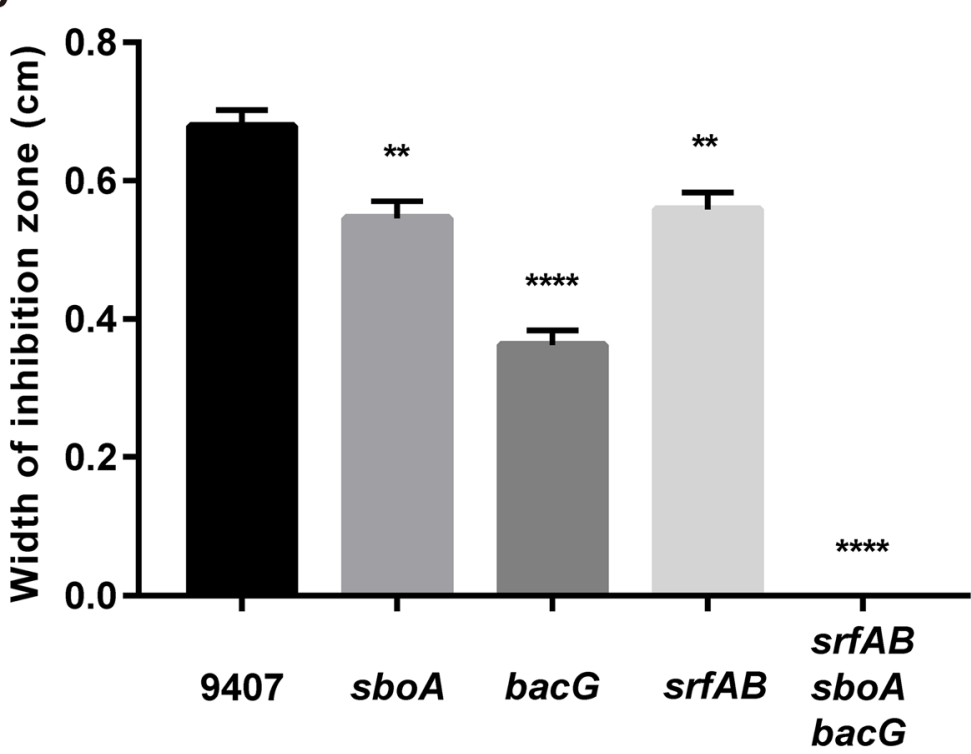

Fig. 4 Antimicrobial activity of the sboA and bacG mutants against $A$. citrulli MH21. a The antimicrobial activity of the sboA and bacG mutants was decreased compared with that of the wild-type B. subtilis 9407 strain. $\mathbf{b}$ The inhibition zone of B. subtilis 9407 , the sboA and bacG mutants against A. citrulli MH21. Antibacterial activity was indicated by the diameter of the inhibition halo minus the diameter of the colony. The statistical analysis was performed using GraphPad Prism 7 software by one-way ANOVA with the Dunnett test $(P<0.05)$. The variation was recorded as mean $\pm \mathrm{SE}\left({ }^{* * * *}: P<0.0001,{ }^{* * *}: P<0.001,{ }^{* *}: P<0.01,{ }^{*}: P<0.05\right)$ 


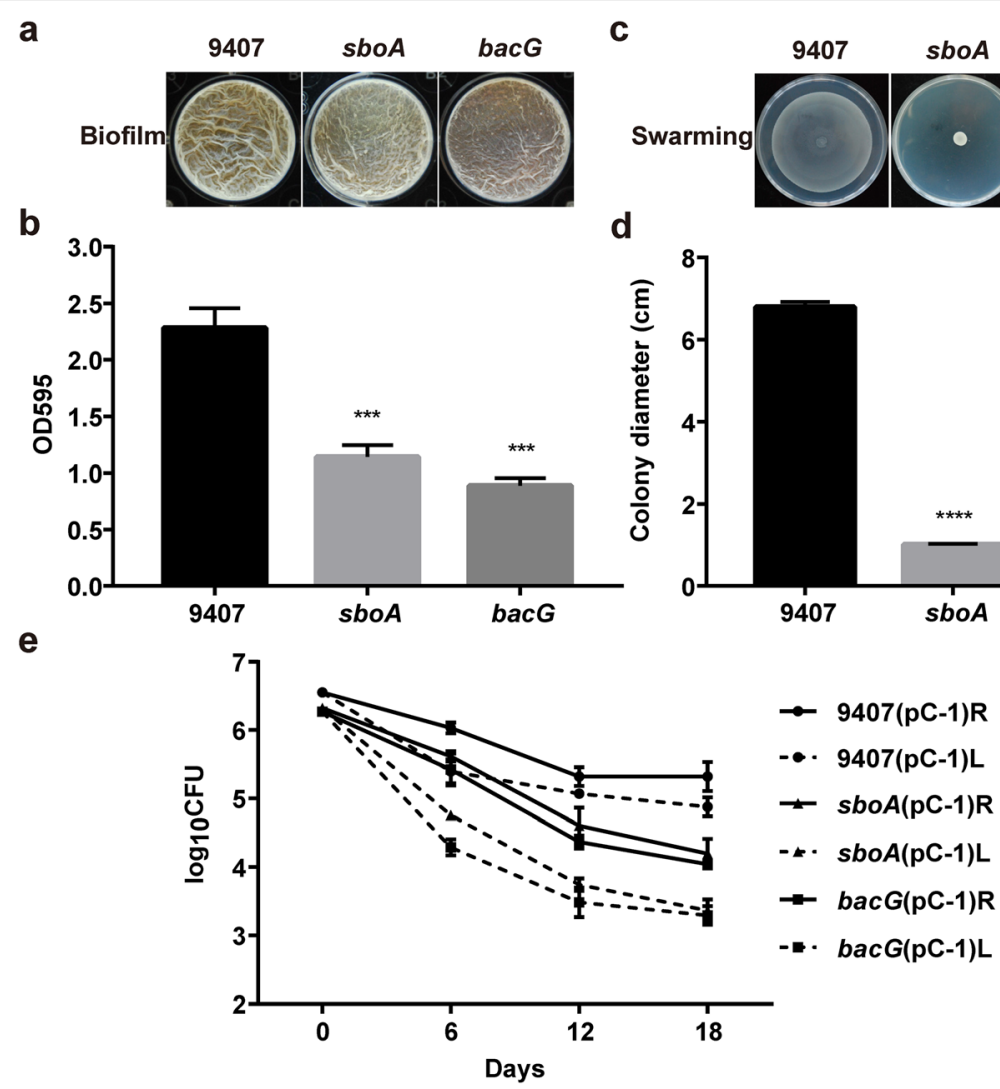

Fig. 5 The impact of deleting sboA or bacG on colonization ability of the B. subtilis 9407 strain. $\mathbf{a}, \mathbf{b}$ Biofilm formation assays. Biofilm assays were detected in Msgg plate incubated at $28^{\circ} \mathrm{C}$ for 96 h. $\mathbf{c}$, d Swarming ability assays. Swarming assays were detected in LA plate (containing $0.7 \%$ agar) incubated at $37^{\circ} \mathrm{C}$ for $6 \mathrm{~h}$. After drying the water, the plates were incubated overnight at $37^{\circ} \mathrm{C}$. e Colonization on the melon roots (R) and leaves $(L)$ of B. subtilis 9407 and mutant strains. To facilitate the screening, all these strains were transformed into a plasmid pC-1 with a chloramphenicol resistance gene. The $y$-axis was $\log _{10} \mathrm{CFU}$ and the $x$-axis was the number of days. The statistical analysis was performed using GraphPad Prism 7 software by one-way ANOVA with the Dunnett test $(P<0.05)$. The variation was recorded as mean $\pm \mathrm{SE}\left({ }^{* * * *}: P<0.0001\right.$, ***: $P<0.001, * *: P<0.01, *: P<0.05)$

this study, we report that B. subtilis 9407 has the potential to colonize plants, inhibit pathogens, promote plant growth, and induce plant systemic resistance; it also produces bacilysin and subtilosin $\mathrm{A}$ in addition to the previously reported surfactin, all of which are active against BFB.

Many studies have shown that swarming motility helps bacteria to migrate to plant roots (Allard-Massicotte et al. 2016; Gao et al. 2016) and that biofilms contribute to plant root colonization (Verstraeten et al. 2008).
Swarming motility relies on a swinging flagellum, encoded by the $f l i$ and $f l g$ operons (Kearns 2010). The eps and tapA-sipW-tasA operons are responsible for synthesizing the main biofilm components, and $\operatorname{spoOA,~} \sin I$, $a b r B, \sin R$ are the main regulatory genes (Verstraeten et al. 2008). The genes $a l b, b a c, p k s, p p s$, and $s r f$ are responsible for the synthesis of antimicrobial substances subtilosin A, bacilysin, bacillaene, fengycin, and surfactin, respectively (Stein 2005; Moldenhauer et al. 2007; Amrouche et al. 2010; Rajavel et al. 2013). The

Table 3 Biocontrol efficacy of the sboA and bacG mutants against BFB was decreased under greenhouse conditions

\begin{tabular}{llll}
\hline Treatment & Disease index & Disease incidence (\%) & Biocontrol effect (\%) \\
\hline Control & $76.7 \pm 4.4 \mathrm{c}$ & $93.3 \pm 5.8 \mathrm{c}$ & 0.0 \\
9407 & $26.1 \pm 2.0 \mathrm{a}$ & $33.3 \pm 5.8 \mathrm{a}$ & 64.3 \\
sboA & $39.4 \pm 1.1 \mathrm{~b}$ & $53.3 \pm 5.8 \mathrm{~b}$ & 42.9 \\
bacG & $47.8 \pm 3.1 \mathrm{~b}$ & $63.3 \pm 5.8 \mathrm{~b}$ & 32.1 \\
\hline
\end{tabular}

Statistical analysis was performed using the SPSS 21.0 software by one-way ANOVA with Tukey's test. Different letters in the columns represent significant differences $(P<0.05)$. The data in the table represent means \pm SE 


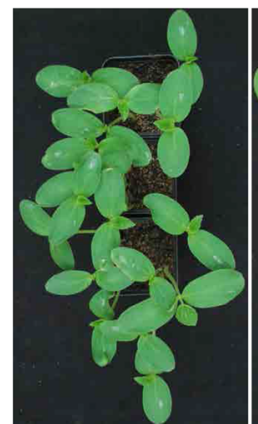

Blank

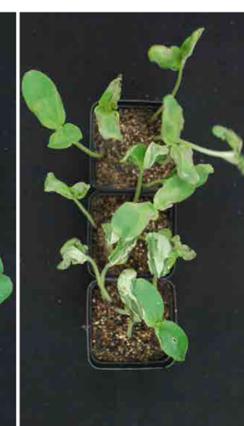

Control

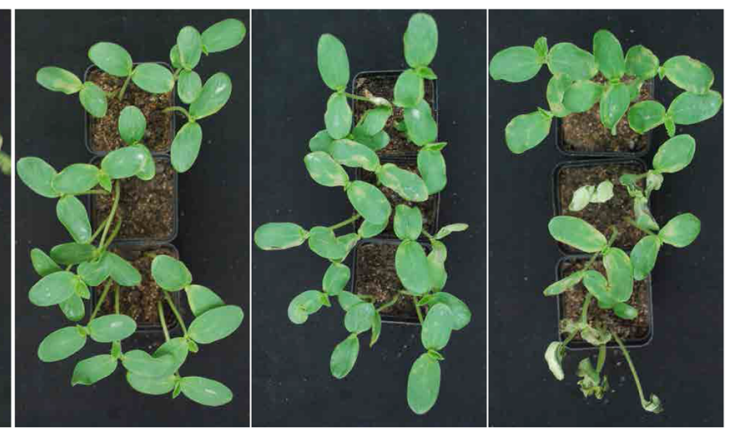

9407
sboA
bacG

Fig. 6 Biocontrol efficacy of the $s b o A$ and bacG mutants against BFB under greenhouse conditions. Blank: PBS buffer was used to immerse the germinated melon seeds, and a saline solution $(0.9 \% \mathrm{NaCl})$ was used to spray the seedlings; Control: PBS buffer was used to immerse the germinated seeds, and the seedlings were sprayed with A. citrulli; 9407: the germinated seeds were soaked in a suspension of B. subtilis 9407 and the seedlings were sprayed with $A$. citrulli; sboA: the germinated seeds were soaked in the sboA mutant and the seedlings were sprayed with $A$. citrulli; bacG: the germinated seeds were soaked in the bacG mutant and the seedlings were sprayed with $A$. citrulli

lipopeptides surfactin and fengycin, which are encoded by $s r f$ and $p p s B$, respectively, are elicitors that induce systemic resistance to protect plants from pathogen infection (Ongena et al. 2007; Wang et al. 2020). The genes related to plant growth promotion, such as $y s n E$, $y w k B$, phyC, dhb operon, and bltD, synthesize IAA, auxin, phytase, siderophore, and spermidine, respectively (Kerovuo et al. 1998; May et al. 2001; Baichoo et al. 2002; Quentin et al. 2002). The presence of these genes in B. subtilis 9407 implies its biocontrol potential for controlling BFB.

The antibacterial compounds subtilosin A and bacilysin, which are produced by Bacillus strains, allows these strains to antagonize pathogens (Khochamit et al. 2015; Wu et al. 2015). B. subtilis KKU213 produces subtilosin A against various gram-positive bacteria and B. amyloliquefaciens FZB42 exhibits biocontrol activity against gram-negative bacterium Xanthomonas strains by producing bacilysin (Khochamit et al. 2015; Wu et al. 2015). Previously, we have determined that surfactin produced by B. subtilis 9407 is crucial for this strain to control BFB (Fan et al. 2017b). In this study, subtilosin A and bacilysin BGCs were identified in the genome of $B$. subtilis 9407 . To verify whether these two compounds play a role in the B. subtilis 9407-mediated control of BFB, we conducted verification tests. We found that the loss of sboA or bacG in B. subtilis 9407 decreased its biocontrol efficacy against BFB by affecting both of its inhibitory activity against $A$. citrulli $\mathrm{MH} 21$ and ability to colonize plant tissues. Subtilosin A, bacilysin, and surfactin showed a synergistic effect on the inhibition of $A$. citrulli MH21. However, the absence of $s b o A$ or bacG slowed the swarming motility and biofilm formation of these strains without affecting their growth (Additional file 1: Figure S4). Surfactin triggers biofilm formation and plant root colonization in B. subtilis, which is crucial for its biocontrol efficacy (Zeriouh et al. 2014). However, there have been no reports of the effects of subtilosin A and bacilysin on biofilm formation and swarming motility. Based on the results of the present study, we speculate that subtilosin $\mathrm{A}$ and bacilysin may have similar functions with surfactin.

\section{Conclusions}

Previous studies showed that B. subtilis 9407 produces surfactin against BFB. Whether B. subtilis 9407 possesses other pathways to control BFB remains unknown at present. This study is the first to report that $B$. subtilis 9407 can control BFB by producing subtilosin A and bacilysin. Subtilosin A and bacilysin contributed to the biocontrol efficacy of B. subtilis 9407 against BFB through their antibacterial activities and plant colonization abilities. Comprehensive genomic analysis of B. subtilis 9407 suggests that this strain still has unrevealed biocontrol mechanism, highlighting its potential as a biocontrol agent. Further research on the biocontrol mechanisms of this beneficial strain will aid the development of biocontrol agents for specific plant diseases.

\section{Methods}

\section{Bacterial growth and construction of mutant strains}

A list containing all strains and plasmids used in this study is presented in Additional file 2: Table S1. A. citrulli MH21 was incubated at $28^{\circ} \mathrm{C}, 200 \mathrm{rpm}$ in $\mathrm{LB}$ broth containing $100 \mu \mathrm{g} / \mathrm{mL}$ of ampicillin.

Deletion mutants of $B$. subtilis were constructed by homologous recombination and screened on LB plates with erythromycin $(5 \mu \mathrm{g} / \mathrm{mL})$, chloramphenicol $(5 \mu \mathrm{g} /$ $\mathrm{mL})$, or kanamycin $(20 \mu \mathrm{g} / \mathrm{mL})$. Briefly, the plasmid containing the resistance cassette flanked by $1 \mathrm{~kb}$ DNA sequences corresponding to the upstream and downstream regions of the target genes was cloned into $E$. coli 
DH5 $\alpha$, and then was introduced into E. coli EC135 without endogenous limiting modification system by chemical conversion method. Finally, the plasmids were introduced into the competent cells of B. subtilis 9407 by electroporation $(1.8 \mathrm{kV}, 200 \Omega, 25 \mu \mathrm{F})$ with a time constant of 4.5 to $5.5 \mathrm{msec}$, and the mutants were obtained by screening with the corresponding antibiotic. In $s b o A$ mutant, the $s b o A$ coding sequence was replaced with a kanamycin resistance cassette. In bacG mutant, the bacG coding sequence was also replaced by a kanamycin resistance cassette. In $\operatorname{srf} A B s b o A b a c G$ mutant, the $\operatorname{srf} A B, s b o A$, and $b a c G$ coding sequences were replaced by the tetracycline, kanamycin, and chloramphenicol resistance cassette, respectively. Transformants were verified by PCR amplification and DNA sequencing.

\section{Genome sequencing, assembly, and annotation}

The draft sequences of the Bacillus strains were produced by using Illumina paired-end sequencing technology at the company of BerryGenomics, Beijing. Assemblies were performed using SOAPdenovo v.2.04 (Luo et al. 2012), resulting in 16 scaffolds for 9407. Predictions of protein-coding genes were implemented using Prokka v.1.11 (Seemann 2014). Functional annotation was carried out using the Basic Local Alignment Search Tool (BLAST) against the Cluster of Orthologous Groups of proteins (COG), NCBI nr protein database, Kyoto Encyclopedia of Genes and Genomes (KEGG) database, and InterPro database. Ordering of contigs of the strain 9407 was achieved using the Java-based graphical interface program Mauve (Rissman et al. 2009). The genome sequence of $B$. subtilis 168 was used as a reference for the strain 9407. The final annotated chromosome was plotted using CIRCOS to show gene locations, GC-skew, and GC content (Krzywinski et al. 2009). A comparative circular genome map was constructed by BRIG v.0.95 to evaluate the synteny of the assembled genome of B. subtilis 9407 with that of B. subtilis 168 (Alikhan et al. 2011).

The whole-genome shotgun data of B. subtilis 9407 have been deposited at GenBank under the accession number PISO00000000.1. The genomic sequence of $B$. subtilis 168 was deposited under the accession number AL009126.3. All of the bacterial strains used in this study and their accession numbers in GenBank are listed in Additional file 2: Table S3.

\section{Phylogenetic analysis of B. subtilis 9407}

All genomes used in this analysis were downloaded in FASTA format from the NCBI database. A maximumlikelihood phylogenetic tree of Bacillus species was constructed based on 662 single-copy core proteins shared by 18 Bacillus genomes and the genome of Paenibacillus polymyxa M1 according to the following methods: (1) multiple alignments of amino acid sequences were carried out by MAFFT v.7.310 (Katoh and Standley 2013); (2) conserved blocks from multiple alignments of test protein were selected by using Gblocks (Castresana 2000); (3) ML tree was constructed using RAxML v.8.2.10 (Stamatakis 2014) software using the PROTGAMMALGX model with 100 bootstrap replicates. The tree was displayed by molecular evolutionary genetic analysis (MEGA) (Kumar et al. 2018). Then, ANI values between two genome sequences were calculated using the original ANI function of OrthoANI (Lee et al. 2016). The heat maps were generated using CIMminer (https://discover.nci.nih.gov/cimminer/) based on ANI values (Scherf et al. 2000). The pan-genomic analysis was performed by the PGAP analysis pipeline (Zhao et al. 2018).

\section{Analysis of functional genes and secondary metabolite biosynthesis gene clusters}

Amino acid sequence identity was compared by the Blastp program between genes of B. subtilis 9407 and 168. The BGCs were predicted using the antiSMASH bacterial v.5.1.2 (Blin et al. 2019) and further analyzed by the 2ndFIND (http:// biosyn.nih.go.jp/2ndfind/) program to confirm more accurate information of BGCs, which was performed via the Web servers with the default parameters.

\section{In vitro antagonism test}

Colonies of $A$. citrulli $\mathrm{MH} 21$ were inoculated into $5 \mathrm{~mL}$ LB broth and incubated on an orbital shaker (200 rpm) until an OD600 of 0.8 was reached, corresponding to a bacterial concentration of approximately $10^{8} \mathrm{CFU} / \mathrm{mL}$. The bacterial suspension was then added to melted and cooled LA medium, mixed and poured into Petri dishes $(9 \mathrm{~cm}$ in diameter), and allowed to re-solidify. Colonies of $B$. subtilis were initially inoculated into $5 \mathrm{~mL}$ of $\mathrm{LB}$ broth and incubated at $37^{\circ} \mathrm{C}$ and $200 \mathrm{rpm}$, for $12 \mathrm{~h}$. The bacteria were then adjusted to an OD600 of $0.8\left(10^{8}\right.$ $\mathrm{CFU} / \mathrm{mL}$ ) using $\mathrm{LB}$ broth and $2 \mu \mathrm{L}$ aliquots of this suspension were added to the surface of the above-prepared LB solid plates. Plates were cultured at $28^{\circ} \mathrm{C}$ for $5 \mathrm{~d}$ and the zone of inhibition was observed and measured. There were five repetitions for each tested B. subtilis strain. The experiments were repeated three times independently.

\section{Biofilm formation assay}

The biofilm formation assay was performed as previously described (Fan et al. 2017b). Colonies of B. subtilis were initially inoculated into $5 \mathrm{~mL}$ of MSgg liquid culture medium ( $5 \mathrm{mM}$ potassium phosphate buffer $\mathrm{pH} 7,100$ $\mathrm{mM}$ Mops pH 7, $2 \mathrm{mM} \mathrm{MgCl}_{2}, 700 \mu \mathrm{M} \mathrm{CaCl}, 50 \mu \mathrm{M}$ $\mathrm{MnCl}_{2}, 50 \mu \mathrm{M} \mathrm{FeCl}_{3}, 1 \mu \mathrm{M} \mathrm{ZnCl}, 2 \mu \mathrm{M}$ thiamine, $0.5 \%$ 
glycerol, $0.5 \%$ glutamate, $50 \mu \mathrm{g} / \mathrm{mL}$ tryptophan, $50 \mu \mathrm{g} /$ $\mathrm{mL}$ phenylalanine), and incubated at $28^{\circ} \mathrm{C}$ and $200 \mathrm{rpm}$ until an OD600 of 0.8 was reached. Then, the bacterial suspension was inoculated into a 12-well microtiter plate (Corning) containing $4 \mathrm{~mL}$ MSgg liquid medium in each well. Four $\mu \mathrm{L}$ aliquots of the bacterial suspension were inoculated into each well. After inoculation, the microtiter plates were incubated statically at $28^{\circ} \mathrm{C}$ for $96 \mathrm{~h}$. Photos were then taken to record the biofilm phenotypes of different strains. For each tested strain, three replicates were included.

The biofilm quantification assay was performed in 96-well polystyrene microplates (Corning) as described previously (Ma et al. 2017). Each well contains $150 \mu \mathrm{L}$ aliquots of MSgg liquid medium, and eight independent replicated wells were used for each tested strain. Then $1.5 \mu \mathrm{L}$ of bacterial suspension were inoculated into each well. After inoculation, the plates were incubated at $28^{\circ} \mathrm{C}$ for $96 \mathrm{~h}$. Then, the bacterial cells were collected separately from each well and washed twice with $200 \mu \mathrm{L}$ of sterile $\mathrm{ddH}_{2} \mathrm{O}$, and stained with $200 \mu \mathrm{L}$ of $0.1 \%$ (w/v) crystal violet (CV) solution. After staining for $15 \mathrm{~min}$, the staining $\mathrm{CV}$ solution was removed, the bacterial cells were washed twice with $200 \mu \mathrm{L}$ sterile $\mathrm{ddH}_{2} \mathrm{O}$ and then $200 \mu \mathrm{L}$ ethanol was used to elute the CV. The optical density of the eluate was measured with a microplate reader (Tecan Infinite F200) at $595 \mathrm{~nm}$. All experiments were repeated three times independently.

\section{Swarming assay}

The swarming assay was performed as previously described (Fan et al. 2017b). B. subtilis 9407 and mutant strains were grown in LB broth until an OD600 of 0.8 was reached. The cells were collected by centrifugation at $5000 \mathrm{rpm}$ and resuspended in PBS buffer $(10 \mathrm{mM}, \mathrm{pH} 7.4)$. For each tested strain, $2 \mu \mathrm{L}$ aliquots of cell suspension were pipetted onto the surface of a LB medium plate $(0.7 \%$ agar, $w / v)$, after that, the plate was incubated at $37^{\circ} \mathrm{C}$ for $4-6 \mathrm{~h}$ to allow the bacteria to swim. Then, the plate was placed in a laminar flow hood with a constant flow of dry air to reduce the water content in the medium and therefore to terminate the swimming process. Subsequently, the plate was incubated overnight at room temperature. Five replicates were included for each strain, and all experiments were repeated three times independently. Photos were taken to record the swarming phenotypes of different strains.

\section{Colonization assay}

The colonization assay was performed in an artificial climate chamber. Melon seeds were incubated in water at $55^{\circ} \mathrm{C}$ for $30 \mathrm{~min}$ and then transferred to a
Petri dish with wet gauze and kept at $28^{\circ} \mathrm{C}$ for $36 \mathrm{~h}$ to allow germination. B. subtilis 9407 and mutant strains were cultured in LB broth to an OD600 of 0.8 . The bacterial cells were collected by centrifugation at $5000 \mathrm{rpm}$ and washed three times with sterile $\mathrm{ddH}_{2} \mathrm{O}$, and then resuspended with PBS buffer. The germinated seeds were soaked in the bacterial suspensions for $30 \mathrm{~min}$ and then sown in pots filled with a mixture of vermiculite and organic soil $(1: 2 \mathrm{v} / \mathrm{v})$, with six seeds per pot. The pots were then placed in an artificial climate chamber at $25^{\circ} \mathrm{C}$ with a $16 \mathrm{~h}$ light and $8 \mathrm{~h}$ dark photoperiod. At 6,12 , and 18 days after sowing, the bacterial population colonizing melon roots and leaves was determined by plate counting as described previously (Fan et al. 2017b). Each straintreated seeds were sown in three pots. The experiment was repeated three times independently.

\section{Evaluation of biocontrol efficacy under greenhouse conditions}

Melon seeds and bacterial suspensions of B. subtilis 9407 and mutant strains were prepared as described above for the colonization assay. Treatment of the germinated seeds with bacterial suspensions of $B$. subtilis 9407 and mutant strains was the same as in the colonization assay. Inoculum of the pathogen $A$. citrulli MH21 was produced in shake culture in 50 $\mathrm{mL} \mathrm{LB}$ broth at $28^{\circ} \mathrm{C}$ for $36 \mathrm{~h}$. The bacterial cells of A. citrulli $\mathrm{MH} 21$ were resuspended in a saline solution $(0.9 \% \mathrm{NaCl})$ and adjusted to an OD600 of 0.8 . Three days after sowing when melon seeds grew two cotyledons, the seedlings were spray-inoculated with A. citrulli MH21. Two controls were included in this experiment. In one control (referred to as "Blank"), PBS buffer instead of B. subtilis 9407, and a saline solution $(0.9 \% \mathrm{NaCl})$ instead of $A$. citrulli were used; in the other control (referred to as "Control"), the germinated seeds were soaked in PBS buffer, and the seedlings were sprayed with $A$. citrulli. Pots with different treatments were randomized across the experimental area in a greenhouse with a light/dark period of $14 / 10 \mathrm{~h}$ at $20-35^{\circ} \mathrm{C}$, and were sprayed with sterilized water every 2 days. Each tested B. subtilis strain had three pots as a repetition, and each pot had six seedlings. Disease incidence and severity were recorded within 3-5 d of the appearance of the first symptoms. The experiment was repeated three times.

Disease severity was rated on a scale from 0 to 6 according to the percentage of symptomatic area in each leaf: 0 , no symptoms; $1,10 \%$ or less symptomatic leaves; 2-5, 11-25\%; 26-50\%; $51-75 \%$ and $76-$ $90 \%$ symptomatic leaves, respectively; and 6, >90\% symptomatic leaves (Bahar et al. 2008). The disease 
index, disease incidence, and disease control effect were calculated as follows:

Disease index $=\left[\sum(\right.$ Rating $\times$ Number of diseased leaves rated $)$

/Total number of leaves $\times$ Highest rating] $\times 100$

Disease incidence $(\%)=($ Total number of diseased leaves

/total number of investigated leaves $) \times 100$

Disease control effect $(\%)=[($ Disease incidence of the control

- Disease incidence of the treatment)

/Disease incidence of the control] $\times 100$

\section{Supplementary Information}

The online version contains supplementary material available at https://doi. org/10.1186/s42483-021-00081-2.

Additional file 1: Figure S1. Genome map of Bacillus subtilis 9407. Figure S2. BRIG based on homology with B. subtilis 168. Figure S3. Schematic diagram of secondary metabolite clusters in the B. subtilis 9407 and 168 genomes. Figure S4. Growth curve of B. subtilis 9407 and mutant strains.

Additional file 2: Table S1. Strains and plasmids used in this study. Table S2. Functional genes involved in biocontrol traits in B. subtilis 9407 and B. subtilis 168 . Table S3. GeneBank accession numbers of the strains used in this study.

\section{Abbreviations}

ANI: Average nucleotide identity; Blast: Basic local alignment search tool; BFB: Bacterial fruit blotch; BGCs: Biosynthesis gene clusters; BRIG: BLAST Ring Image Generator; COG: Cluster of Orthologous Groups of proteins; IAA: Indole-3-acetic acid; KEGG: Kyoto Encyclopedia of Genes and Genomes; ML: Maximum likelihood; NCBI: National Center for Biotechnology Information; ORF: Open reading frame; VOCs: Volatile organic compounds

\section{Acknowledgments}

We are thankful to Prof. Liqun Zhang of College of Plant Protection, China Agricultural University for providing A. citrulli $\mathrm{MH} 21$ strain.

\section{Authors' contributions}

XG wrote the manuscript and performed the experiments. QZ, XG, and $Y Z$ analyzed the complete genomic sequence of $B$. subtilis $9407 . X G, Y W$, and $J L$ discussed the results. QW and $Y L$ helped with the design of the experiments and revised the manuscript. All authors read and approved the final manuscript.

\section{Funding}

This work was supported by Major Science and Technology Innovation Projects in Shandong Province (2019JZZY010718) and National Key Research and Development Project (2019YFD1002003)

\section{Availability of data and materials}

Not applicable.

Ethics approval and consent to participate

Not applicable.

\section{Consent for publication}

Not applicable.

\section{Competing interests}

The authors declare no conflict of interest.

\section{Author details}

'Department of Plant Pathology, MOA Key Lab of Pest Monitoring and Green Management, College of Plant Protection, China Agricultural University,
Beijing 100193, China. ${ }^{2}$ Beijing Advanced Innovation Center for Tree Breeding by Molecular Design, Beijing Forestry University, Beijing 100083, China. ${ }^{3}$ Ordos Plant Protection and Quarantine Station, Ordos 017000, China. ${ }^{4}$ Ecology Institute, Qilu University of Technology (Shandong Academy of Sciences), Jinan 250014, China.

Received: 24 September 2020 Accepted: 19 January 2021

Published online: 03 February 2021

\section{References}

Adhikari M, Yadav DR, Kim SW, Um YH, Kim HS, Lee SC, et al. Biological control of bacterial fruit blotch of watermelon pathogen (Acidovorax citrulli) with rhizosphere associated bacteria. Plant Pathol J. 2017;33:170-83.

Al-Ali A, Deravel J, Krier F, Bechet M, Ongena M, Jacques P. Biofilm formation is determinant in tomato rhizosphere colonization by Bacillus velezensis FZB42. Environ Sci Pollut Res Int. 2018;25:29910-20.

Alanjary M, Medema MH. Mining bacterial genomes to reveal secret synergy. J Biol Chem. 2018:293:19996-7.

Alikhan NF, Petty NK, Ben Zakour NL, Beatson SA. BLAST ring image generator (BRIG): simple prokaryote genome comparisons. BMC Genomics. 2011;12:402.

Allard-Massicotte R, Tessier L, Lecuyer F, Lakshmanan V, Lucier JF, Garneau D, et al. Bacillus subtilis early colonization of Arabidopsis thaliana roots involves multiple chemotaxis receptors. mBio. 2016;7:e01664-16.

Amrouche T, Sutyak Noll K, Wang Y, Huang Q, Chikindas ML. Antibacterial activity of subtilosin alone and combined with curcumin, poly-lysine and zinc lactate against Listeria monocytogenes strains. Probiotics Antimicrob Proteins. 2010;2: 250-7

Ashwini N, Srividya S. Potentiality of Bacillus subtilis as biocontrol agent for management of anthracnose disease of chilli caused by Colletotrichum gloeosporioides OGC1. 3 Biotech. 2014;4:127-36.

Bahar O, Kritzman G, Burdman S. Bacterial fruit blotch of melon: screens for disease tolerance and role of seed transmission in pathogenicity. Eur J Plant Pathol. 2008;123:71-83.

Baichoo N, Wang T, Ye R, Helmann JD. Global analysis of the Bacillus subtilis Fur regulon and the iron starvation stimulon. Mol Microbiol. 2002;45: 1613-29.

Barbe V, Cruveiller S, Kunst F, Lenoble P, Meurice G, Sekowska A, et al. From a consortium sequence to a unified sequence: the Bacillus subtilis 168 reference genome a decade later. Microbiology. 2009:155:1758-75.

Blin K, Shaw S, Steinke K, Villebro R, Ziemert N, Lee SY, et al. antiSMASH 5.0: updates to the secondary metabolite genome mining pipeline. Nucleic Acids Res. 2019;47:W81-7.

Castresana J. Selection of conserved blocks from multiple alignments for their use in phylogenetic analysis. Mol Biol Evol. 2000;17:540-52.

Fan H, Ru J, Zhang Y, Wang Q, Li Y. Fengycin produced by Bacillus subtilis 9407 plays a major role in the biocontrol of apple ring rot disease. Microbiol Res. 2017a;199:89-97.

Fan H, Zhang Z, Li Y, Zhang X, Duan Y, Wang Q. Biocontrol of bacterial fruit blotch by Bacillus subtilis 9407 via surfactin-mediated antibacterial activity and colonization. Front Microbiol. 2017b;8:1973.

Fira D, Dimkic I, Beric T, Lozo J, Stankovic S. Biological control of plant pathogens by Bacillus species. J Biotechnol. 2018;285:44-55.

Franco-Sierra ND, Posada LF, Santa-Maria G, Romero-Tabarez M, Villegas-Escobar V. Alvarez JC. Bacillus subtilis EA-CB0575 genome reveals clues for plant growth promotion and potential for sustainable agriculture. Funct Integr Genomics. 2020:20:575-89.

Gao S, Wu H, Wang W, Yang Y, Xie S, Xie Y, et al. Efficient colonization and harpins mediated enhancement in growth and biocontrol of wilt disease in tomato by Bacillus subtilis. Lett Appl Microbiol. 2013;57:526-33.

Gao S, Wu H, Yu X, Qian L, Gao X. Swarming motility plays the major role in migration during tomato root colonization by Bacillus subtilis SWRO1. Bio Control. 2016;98:11-7.

Hashem A, Tabassum B, Abd_Allah EF. Bacillus subtilis: A plant-growth promoting rhizobacterium that also impacts biotic stress. Saudi J Biol Sci. 2019;26:1291-7.

Jiang CH, Wu F, Yu ZY, Xie P, Ke HJ, Li HW, et al. Study on screening and antagonistic mechanisms of Bacillus amyloliquefaciens 54 against bacterial fruit blotch (BFB) caused by Acidovorax avenae subsp. citrulli. Microbiol Res. 2015;170:95-104.

Katoh K, Standley DM. MAFFT multiple sequence alignment software version 7 : improvements in performance and usability. Mol Biol Evol. 2013;30:772-80. 
Kearns DB. A field guide to bacterial swarming motility. Nat Rev Microbiol. 2010;8: 634-44.

Kerovuo J, Lauraeus M, Nurminen P, Kalkkinen N, Apajalahti J. Isolation, characterization, molecular gene cloning, and sequencing of a novel phytase from Bacillus subtilis. Appl Environ Microbiol. 1998;64:2079-85.

Khochamit N, Siripornadulsil S, Sukon P, Siripornadulsil W. Antibacterial activity and genotypic-phenotypic characteristics of bacteriocinproducing Bacillus subtilis KKU213: potential as a probiotic strain. Microbiol Res. 2015;170:36-50.

Koumoutsi A, Chen XH, Henne A, Liesegang H, Hitzeroth G, Franke P, et al. Structural and functional characterization of gene clusters directing nonribosomal synthesis of bioactive cyclic lipopeptides in Bacillus amyloliquefaciens strain FZB42. J Bacteriol. 2004;186:1084-96.

Krzywinski M, Schein J, Birol I, Connors J, Gascoyne R, Horsman D, et al. Circos: an information aesthetic for comparative genomics. Genome Res. 2009;19:1639-45.

Kumar S, Stecher G, Li M, Knyaz C, Tamura K. MEGA X: Molecular evolutionary genetics analysis across computing platforms. Mol Biol Evol. 2018;35:1547-9.

Kunst F, Ogasawara N, Moszer I, Albertini AM, Alloni G, Azevedo V, et al. The complete genome sequence of the gram-positive bacterium Bacillus subtilis. Nature. 1997;390:249-56.

Lee I, Ouk Kim Y, Park SC, Chun J. OrthoANI: an improved algorithm and software for calculating average nucleotide identity. Int J Syst Evol Microbiol. 2016;66: 1100-3.

Luo R, Liu B, Xie Y, Li Z, Huang W, Yuan J, et al. SOAPdenovo2: an empirically improved memory-efficient short-read de novo assembler. GigaScience. 2012;1:18.

Ma W, Peng D, Walker SL, Cao B, Gao CH, Huang Q, et al. Bacillus subtilis biofilm development in the presence of soil clay minerals and iron oxides. NPJ Biofilms Microbiomes. 2017;3:4.

May JJ, Wendrich TM, Marahiel MA. The dhb operon of Bacillus subtilis encodes the biosynthetic template for the catecholic siderophore 2,3dihydroxybenzoate-glycine-threonine trimeric ester bacillibactin. J Biol Chem. 2001;276:7209-17.

Moldenhauer J, Chen XH, Borriss R, Piel J. Biosynthesis of the antibiotic bacillaene, the product of a giant polyketide synthase complex of the trans-AT family. Angew Chem Int Ed Engl. 2007;46:8195-7.

Moszer I. The complete genome of Bacillus subtilis: from sequence annotation to data management and analysis. FEBS Lett. 1998;430:28-36.

Ongena M, Jourdan E, Adam A, Paquot M, Brans A, Joris B, et al. Surfactin and fengycin lipopeptides of Bacillus subtilis as elicitors of induced systemic resistance in plants. Environ Microbiol. 2007;9:1084-90.

Parks DH, Chuvochina M, Waite DW, Rinke C, Skarshewski A, Chaumeil PA, et al. A standardized bacterial taxonomy based on genome phylogeny substantially revises the tree of life. Nat Biotechnol. 2018;36:996-1004.

Quentin Y, Chabalier J, Fichant G. Strategies for the identification, the assembly and the classification of integrated biological systems in completely sequenced genomes. Comput Chem. 2002;26:447-57.

Rahimi T, Niazi A, Deihimi T, Taghavi SM, Ayatollahi S, Ebrahimie E. Genome annotation and comparative genomic analysis of Bacillus subtilis MJ01, a new bio-degradation strain isolated from oil-contaminated soil. Funct Integr Genomics. 2018;18:533-43.

Rahimi-Midani A, Choi TJ. Transport of phage in melon plants and inhibition of progression of bacterial fruit blotch. Viruses. 2020;12:477.

Rajavel M, Perinbam K, Gopal B. Structural insights into the role of Bacillus subtilis YwfH (BacG) in tetrahydrotyrosine synthesis. Acta Crystallogr Sect D: Struct Biol. 2013;69:324-32.

Rissman Al, Mau B, Biehl BS, Darling AE, Glasner JD, Perna NT. Reordering contigs of draft genomes using the mauve aligner. Bioinformatics. 2009;25:2071-3.

Santos ER, Gouveia ER, Mariano RLR, Souto-Maior AM. Controle biológico da mancha-aquosa do melão por compostos bioativos produzidos por Bacillus spp. Summa Phytopathol. 2006;32:376-8.

Scherf U, Ross DT, Waltham M, Smith LH, Lee JK, Tanabe L, et al. A gene expression database for the molecular pharmacology of cancer. Nat Genet. 2000;24:236-44

Seemann T. Prokka: rapid prokaryotic genome annotation. Bioinformatics. 2014; 30:2068-9.

Stamatakis A. RAxML version 8: a tool for phylogenetic analysis and post-analysis of large phylogenies. Bioinformatics. 2014;30:1312-3.

Stein T. Bacillus subtilis antibiotics: structures, syntheses and specific functions. Mol Microbiol. 2005;56:845-57.
Sulthana A, Lakshmi SG, Madempudi RS. Genome sequencing and annotation of Bacillus subtilis UBBS-14 to ensure probiotic safety. J Genomics. 2019;7:14-7.

Verstraeten N, Braeken K, Debkumari B, Fauvart M, Fransaer J, Vermant J, et al. Living on a surface: swarming and biofilm formation. Trends Microbiol. 2008; 16:496-506.

Wang Y, Zhang C, Liang J, Wang L, Gao W, Jiang J, et al. Surfactin and fengycin B extracted from Bacillus pumilus W-7 provide protection against potato late blight via distinct and synergistic mechanisms. Appl Microbiol Biotechnol. 2020;104:7467-81.

Wu L, Wu H, Chen L, Yu X, Borriss R, Gao X. Difficidin and bacilysin from Bacillus amyloliquefaciens FZB42 have antibacterial activity against Xanthomonas oryzae rice pathogens. Sci Rep. 2015;5:12975.

Zeriouh H, de Vicente A, Perez-Garcia A, Romero D. Surfactin triggers biofilm formation of Bacillus subtilis in melon phylloplane and contributes to the biocontrol activity. Environ Microbiol. 2014;16:2196-211.

Zhao Y, Sun C, Zhao D, Zhang Y, You Y, Jia X, et al. PGAP-X: extension on pangenome analysis pipeline. BMC Genomics. 2018;19:36.

Zheng G, Hehn R, Zuber P. Mutational analysis of the sbo-alb locus of Bacillus subtilis: identification of genes required for subtilosin production and immunity. J Bacteriol. 2000;182:3266-73.

\section{Ready to submit your research? Choose BMC and benefit from:}

- fast, convenient online submission

- thorough peer review by experienced researchers in your field

- rapid publication on acceptance

- support for research data, including large and complex data types

- gold Open Access which fosters wider collaboration and increased citations

- maximum visibility for your research: over $100 \mathrm{M}$ website views per year

At BMC, research is always in progress.

Learn more biomedcentral.com/submissions 